\title{
TEM studies of Ge nanocrystal formation in PECVD grown $\mathrm{SiO}_{2}: \mathrm{Ge} / \mathrm{SiO}_{2}$ multilayers
}

To cite this article: S Aan et al 2006 J. Phys.: Condens. Matter 185037

View the article online for updates and enhancements.

\section{Related content}
Influence of reductant and germanium concentration on the growth and stress developmentof germanium nanocrystals in silicon oxide matrix H G Chew, F Zheng, W K Choi et al.
- Effect of germanium concentration and oxide diffusion barrier on the formation anddistribution of germanium nanocrystals in silicon oxide matrix H G Chew, W K Choi, Y L Foo et al
- Effect of high temperature annealing on the charge trapping characteristics of
silicon nanocrystals embeddedwithin $\mathrm{SiO}$ V loannou-Sougleridis, A G Nassiopoulou and A Travlos

\section{Recent citations}

- Infrared luminescence of annealed
$\frac{\text { germanosilicate Lavers }}{\text { M.S. Tokay et al }}$
- Dense Ge nanocrystal lavers embedded in
$\frac{\text { oxide obtained by controlling the }}{\text { diffusion-crystallization process }}$
Ana-Maria Lepadatu et al
- Composition and structure of Pd
$\frac{\text { nanoclusters in SiOx thin film }}{\text { A. Thogersen et al }}$

\section{IOP ebooks}

Bringing you innovative digital publishing with leading voices to create your essential collection of books in STEM research. Start exploring the collection - download the first chapter of every title for free. 


\title{
TEM studies of Ge nanocrystal formation in PECVD grown $\mathrm{SiO}_{2}: \mathrm{Ge} / \mathrm{SiO}_{2}$ multilayers
}

\author{
S Ağan ${ }^{1}$, A Dana ${ }^{2}$ and A Aydınlı ${ }^{2}$ \\ ${ }^{1}$ Physics Department, Kırıkkale University, 71450 Kırıkkale, Turkey \\ 2 Physics Department, Bilkent University, 06800 Ankara, Turkey \\ Received 16 January 2006 \\ Published 16 May 2006 \\ Online at stacks.iop.org/JPhysCM/18/5037
}

\begin{abstract}
We investigate the effect of annealing on the Ge nanocrystal formation in multilayered germanosilicate-oxide films grown on $\mathrm{Si}$ substrates by plasma enhanced chemical vapour deposition (PECVD). The multilayered samples were annealed at temperatures ranging from 750 to $900^{\circ} \mathrm{C}$ for 5 min under nitrogen atmosphere. The onset of formation of Ge nanocrystals, at $750^{\circ} \mathrm{C}$, can be observed via high resolution TEM micrographs. The diameters of Ge nanocrystals were observed to be between 5 and $14 \mathrm{~nm}$. As the annealing temperature is raised to $850^{\circ} \mathrm{C}$, a second layer of Ge nanocrystals forms next to the original precipitation band, positioning itself closer to the substrate $\mathrm{SiO}_{2}$ interface. High resolution cross section TEM images, electron diffraction and electron energy-loss spectroscopy as well as energy-dispersive $\mathrm{x}$-ray analysis $(\mathrm{EDAX})$ data all indicate that Ge nanocrystals are present in each layer.
\end{abstract}

\section{Introduction}

Recently, different techniques have been tried to fabricate quantum-size particles of semiconductors. The information about structural and morphological characteristics of these semiconductor nanocrystals is very important for modelling electronic properties and designing the structure of optoelectronic devices. The potential applications of the Ge nanocrystals have attracted much attention due to optical and electronic properties, which may be photodetectors [1], light emitters [2], single electron transistors [3], photonic structures [4], quantum computers [5], non-linear optical media [6] and photosensitive materials [7]. In particular, embedding silicon, germanium or silicon-germanium nanocrystals in an insulator matrix has been proposed for non-volatile memory devices [8]. As charge loss through lateral paths in nanocrystal based memory devices is suppressed by the oxide isolation between nanocrystals, these devices exhibit superior charge retention characteristics compared with conventional floating-gate memory devices [9-11]. Retention time in a quantum dot memory device is reported to be strongly related to the shape and the size of the nanocrystals [7]. Retention time improvement of an aligned Si double layer nanocrystal structure has also been reported [12] by scaling the lower layer nanocrystal size. Even though the stacked nanocrystals are not exactly aligned, the lower layer nanocrystals can still help to reduce charge leakage 
from the upper layer nanocrystals [13]. Finally, further increases in memory density may be envisioned through multilayered structures.

The key issue, therefore, in the growth process is to synthesize Ge nanocrystals in an insulating matrix, preferably in the form of thin films, with a narrow size distribution and low defect density. For memory device applications, it is also crucial to control the thickness of the $\mathrm{SiO}_{2}$ tunnel oxide underneath the nanocrystal layer, as well as the density and size of the Ge nanocrystals. While a number of techniques to prepare Ge nanocrystals are being used, germanosilicate films deposited by PECVD have some advantages due to low temperature of deposition, excellent step coverage characteristics, high blocking effects against moisture and alkaline ions, and relatively high dielectric constant values [14]. The direct growth process is probably the most promising among the techniques that have been proposed. PECVD is also very suitable for growth of multilayers with differing compositions. TEM is a powerful technique for the structural and chemical investigation of a wide range of materials. It provides direct evidence of phase separation and crystallization, as well as crystal size and distribution. The ability to determine the size and structure of nanoparticles would provide information to allow the fabrication of structures with desired electrical and optoelectronic properties for device applications.

TEM has, previously, been used for structural analysis of Ge nanocrystals fabricated by cosputtering and annealing $[15,16]$. Cross sectional TEM images provided direct evidence of the nanocrystal formation. It has been observed that lower Ge content results in smaller Ge nanocrystal size. Similar TEM studies of Ge nanocrystals have been performed on samples prepared by the ion implantation technique [17]. With increasing annealing time, it has been observed that the mean density of Ge nanocrystals decreases while the average size of the nanocrystals increases. Choi et al have also reported results of TEM studies of Ge nanocrystals obtained by rapid thermal annealing of cosputtered $\left(\mathrm{Ge}\right.$ and $\left.\mathrm{SiO}_{2}\right)$ samples [18]. At $900{ }^{\circ} \mathrm{C}$ they have observed two regions with different nanocrystal densities. At higher temperatures nanocrystals were observed to form only at the $\mathrm{Si} / \mathrm{SiO}_{2}$ interface. We have previously studied Ge nanocrystals using TEM in single layers grown by PECVD [19] and post-annealing. At an annealing temperature of $1010^{\circ} \mathrm{C}$, we found that smaller crystals form at the $\mathrm{Si} / \mathrm{SiO}_{2}$ interface while larger size crystals form away from the interface.

In the literature, $\mathrm{Si}$ nanocrystals in $\mathrm{Si} / \mathrm{SiO}_{2}$ multilayers were obtained by a lowpressure chemical vapour deposition (LPCVD) of thin silicon layers and atmospheric pressure chemical vapour deposition (APCVD) of $\mathrm{SiO}_{2}$ layers followed by high-temperature thermal oxidation [20, 21]. Molecular beam epitaxy [22] and electron beam deposition [23] have also been used. However, there is no report of Ge nanocrystal formation in $\mathrm{SiO}_{2}$ multilayers in the literature.

In this work, we investigate the effect of annealing on the Ge nanocrystal formation in multilayered germanosilicate-oxide multilayers grown on $\mathrm{Si}$ substrates by plasma enhanced chemical vapour deposition (PECVD). The multilayered samples were annealed at temperatures ranging from 750 to $900{ }^{\circ} \mathrm{C}$ for 5 min under nitrogen atmosphere. The formation and size of $\mathrm{Ge}$ nanocrystals were determined from high resolution electron microscopic (HREM) observations. The samples for the cross-sectional HREM observations were prepared by standard procedures including mechanical and low temperature $(200 \mathrm{~K})$ Ar-ion milling techniques with care taken to minimize radiation damage due to impacting $\mathrm{Ar}^{+}$ions.

\section{Experimental procedure}

The multi-layered structures, with different annealing temperatures, from 750 to $900{ }^{\circ} \mathrm{C}$, were prepared. The oxide $(10 \mathrm{~nm})$-germanosilicate $(20 \mathrm{~nm})$-oxide $(30 \mathrm{~nm})$ multi-layered films were 
grown in a PECVD reactor (model PlasmaLab 8510C) on Si substrates using $180 \mathrm{sccm} \mathrm{SiH}_{4}$ ( $2 \%$ in $\mathrm{N}_{2}$ ), $225 \mathrm{sccm} \mathrm{NO}_{2}$ and varying flow rates of $\mathrm{GeH}_{4}(2 \%$ in $\mathrm{He})$ as precursor gases, at a sample temperature of $350^{\circ} \mathrm{C}$, a process pressure of $1000 \mathrm{mTorr}$ under and an applied RF power of $10 \mathrm{~W}$. First, a tunnel oxide followed by a deposition of a $\mathrm{SiO}_{2}:$ Ge layer and a layer of $\mathrm{SiO}_{2}$ layer was grown. $\mathrm{SiO}_{2}$ : Ge and $\mathrm{SiO}_{2}$ layers form a pair. Samples with layers ranging from three to 10 pairs have been grown. The germanium doped $\mathrm{SiO}_{2}:$ Ge layer is where nanocrystals form upon annealing. Films were grown on p-type silicon substrates with resistivity of $55 \Omega \mathrm{cm}$. The samples were then annealed in $\mathrm{N}_{2}$ atmosphere in a quartz oven at temperatures ranging from 750 to $900^{\circ} \mathrm{C}$ for $5 \mathrm{~min}$. The samples were loaded and unloaded in ramp times of $1 \mathrm{~min}$.

Samples for TEM observation were prepared in cross-section orientation, so that the film layers were viewed edge-on. This preserves the information about the position of the nanocrystals with respect to the surface. The structural characterization was carried out in a JEOL 2010F field-emission transmission electron microscope at $200 \mathrm{kV}$ making use of diffraction, tilt and rotation facilities.

\section{Results and discussion}

TEM studies of several samples were carried out at $200 \mathrm{kV}$. After a brief survey of Ge precipitation, several clusters in each sample were studied for structural detail using high resolution selective area diffraction. High resolution scanning TEM images are formed in the JEOL $2010 \mathrm{~F}$ by focusing coherent electrons into a probe and scanning mode across the sample.

Most of the data presented in this work are obtained on samples with a composition of $\mathrm{Si}_{1.0} \mathrm{Ge}_{0.54} \mathrm{O}_{3.4}$ as determined by $\mathrm{x}$-ray photoelectron spectroscopy where the germane flow rate during deposition was $90 \mathrm{sccm}$. Multilayers of up to 10 pairs have been grown. The multilayered samples were later annealed at temperatures ranging from 750 to $900^{\circ} \mathrm{C}$ for 5 min in nitrogen atmosphere. TEM observations indicate that the Ge nanocrystals can be arranged at a specific depth and for a specific number of layers with nanometre precision.

In figure 1(a), bright field images of samples with three pairs of $\mathrm{SiO}_{2} / \mathrm{SiO}_{2}$ :Ge layers annealed at $750{ }^{\circ} \mathrm{C}$ obtained via TEM are shown as an example. We observe the formation of three dark bands separated by light bands of oxide, indicating diffusion and precipitation of Ge atom clusters in each $\mathrm{Ge}$ doped $\mathrm{SiO}_{2}$ layer. It is seems from figure 1(a) that the initial growth rates of both the oxide layer and the germanium doped layer are smaller than the following layers. A high resolution image of the same sample is shown in figure 1(b). We find that crystallization of Ge clusters has started and crystal planes are observable. Crystallized Ge forms a quasi-continuous layer with crystal planes interwoven with defects. Crystal sizes are on the order of $5 \mathrm{~nm}$. The amorphous character of the surrounding $\mathrm{SiO}_{2}$ matrix as well as the crystal planes of the silicon substrate is also observable. We have taken electron diffraction data from the same area of the sample, shown in figure 1(c). Diffraction data indicate that there are a lot of defects in the Ge layers. We find that these samples are at the onset of crystallization for the Ge clusters. Similar TEM data of other samples annealed at $800,825,850$ and $900{ }^{\circ} \mathrm{C}$ show that as the anneal temperature increases the defect density in the nanocrystals decreases and the size of the nanocrystals increases. Figure 2 illustrates the progress of crystallization further. The sample in figure 2 is annealed at $825^{\circ} \mathrm{C}$. We see a combination of crystallized and amorphous germanium clusters. The average sizes of the crystallized Ge are $8 \mathrm{~nm}$. We clearly observe a twin boundary in the lower Ge nanocrystal. However, the Ge nanocrystal in the upper part of the micrograph shows perfect crystallization without any defects. Observation of perfect crystal planes for the Si substrate shows the high quality of the TEM images. A small cluster in between two nanocrystals does not show crystallization. This indicates that all Ge clusters are not crystallized at this annealing temperature and time. We also note the absence of 

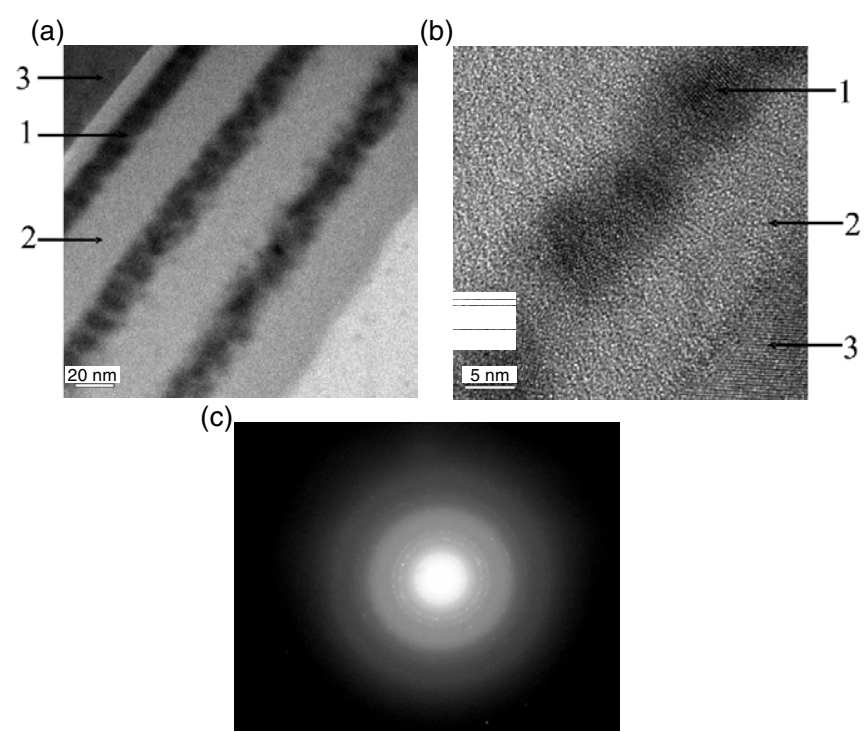

Figure 1. Bright field TEM micrograph of a three pair sample annealed at $750{ }^{\circ} \mathrm{C}$ for $5 \mathrm{~min}$. The samples were grown with $90 \mathrm{sccm}$ of germane flow rate. Ge clusters are observed as dark bands separated by $\mathrm{SiO}_{2}$ layers (a) and exhibit crystal planes confirming the formation of nanocrystals when studied under high resolution (b). In (a) we point to Ge clusters (1), $\mathrm{SiO}_{2}$ spacer layers (2), and Si substrate (3). The electron diffraction pattern of the same sample exhibits hazy rings superimposed on a diffraction pattern indicating the presence of a high density of defects.

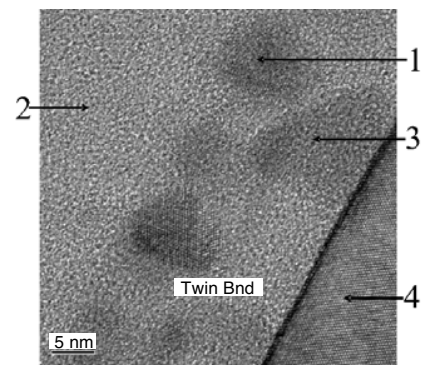

Figure 2. Cross section TEM micrograph of a sample annealed at $825^{\circ} \mathrm{C}$ for $5 \mathrm{~min}$. The samples were grown with $90 \mathrm{sccm}$ of germane flow rate. Twin boundary defects in Ge nanocrystals (1), the oxide matrix (2), Cu contamination from the sample grid (3) and the Si substrate (4) are indicated.

nanocrystals in the near interface for a narrow band of oxide layer. The dark irregular patch (3) in figure 2 is $\mathrm{Cu}$ contamination that has been identified by EDAX analysis near the Si substrate (4) interface. This may be due to the $\mathrm{Cu}$ grid used to hold the sample during the ion beam milling process.

In order to study the influence of Ge concentration in the as grown material on the crystallization of Ge nanocrystals, we have also prepared samples grown with germane flow rate of $120 \mathrm{sccm}$. These films have a composition of $\mathrm{Si}_{1.0} \mathrm{Ge}_{0.67} \mathrm{O}_{3.6}$. The high resolution TEM micrograph of these samples is shown in figure 3. The oxide matrix as well as a continuous band of Ge clusters is clearly observed. The Ge nanocrystal planes are well resolved. Due to excess Ge all the nanocrystals are connected. A number of twin boundaries separate various 


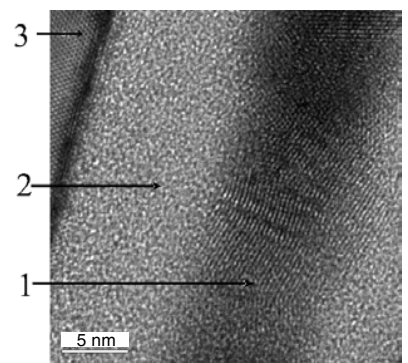

Figure 3. Cross section TEM micrograph of a sample grown with $120 \mathrm{sccm}$ of germane flow rate and annealed at $800^{\circ} \mathrm{C}$ for $5 \mathrm{~min}$. Higher Ge concentration results in continuous distribution of Ge crystal planes (1) with locally changing planar directions surrounded by the oxide matrix (2) and the Si substrate (3).
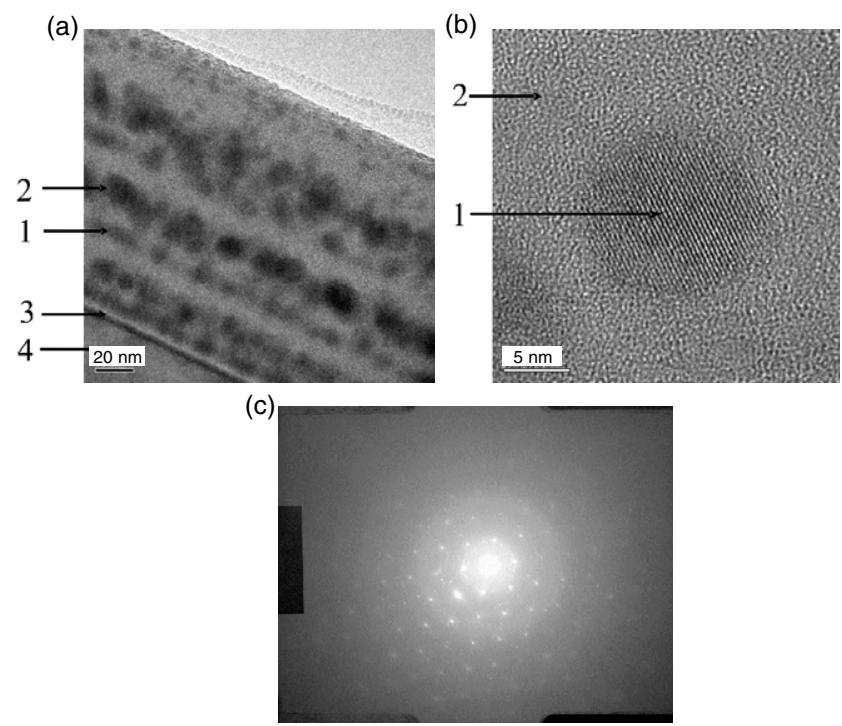

Figure 4. Bright field TEM cross section micrographs with Ge nanocrystals for samples grown with $90 \mathrm{sccm}$ Ge flow rate and annealed at $900^{\circ} \mathrm{C}$ for $5 \mathrm{~min}$. Small (1) and large Ge nanocrystals (2) as well as the thin layer of oxide at the interface (3) are indicated. High resolution TEM study of the same sample shows perfectly aligned crystal planes surrounded by the oxide matrix. Electron beam diffraction patterns confirm excellent crystallinity of the same nanocrystal (c).

nanocrystals from each other. Ge nanocrystal layers are separated from each other with $\mathrm{SiO}_{2}$ layers. We feel that multiple buried epitaxial Ge crystal layers may be obtained from these samples grown with high Ge concentration in the oxide matrix, under optimized processing conditions.

As an example of full crystallization with no observable defects, we show a bright field TEM micrograph of the sample grown with $90 \mathrm{sccm}$ of germane flow rate and annealed at $900{ }^{\circ} \mathrm{C}$ in figure 4 . The sample contains three pairs of $\mathrm{SiO}_{2}: \mathrm{Ge}_{\mathrm{SiO}}$ layers. Dark patches are $\mathrm{Ge}$ nanocrystals in the surrounding $\mathrm{SiO}_{2}$ matrix. It is clearly seen that as the annealing temperature increases, two distinct layers of Ge nanocrystals form in each Ge doped layer. One of the layers is composed of relatively large nanocrystals, whereas the other layer is composed 
of smaller size nanocrystals. The bilayer formation starts above $850{ }^{\circ} \mathrm{C}$ in the samples studied. The smaller size nanocrystals are formed closer to Si substrate followed by larger nanocrystals. TEM micrographs suggest that the smaller Ge nanocrystals evolve from the larger nanocrystals and diffuse and expand into the oxide layers separating the germanosilicate layers (figure 4(a)).

A high resolution TEM image of a typical nanocrystal from the same sample annealed at $900{ }^{\circ} \mathrm{C}$ for $5 \mathrm{~min}$ is shown in figure 4(b). This illustrates an example of full crystallization in a nanocrystal size of $14 \mathrm{~nm}$ in diameter with no observable defects. High resolution micrographs and selective area diffraction confirm that perfect Ge nanocrystals are formed in these samples. It can clearly be seen that Ge nanocrystals have well defined spherical shapes. The size of the crystal islands can be determined from the TEM images. It should be noted that Ge nanocrystal size increases with increasing annealing temperatures. The micrograph shows perfect alignment of crystallographic planes in the Ge nanocrystal that has clearly pronounced facets. The average separation between individual Ge nanocrystals is about $4 \mathrm{~nm}$, corresponding to the nanocrystal density.

In figure 4(c), the crystallinity of the islands was further confirmed by selective area electron diffraction of Ge nanocrystals for a representative sample. We have obtained excellent diffraction patterns compared with those obtained from the samples annealed at $750{ }^{\circ} \mathrm{C}$. This indicates that at $900^{\circ} \mathrm{C}$ full crystallization of Ge clusters takes place. These Ge nanocrystals do not exhibit any defects. It is interesting to note that the crystal quality of the sample annealed at $900{ }^{\circ} \mathrm{C}$ for $5 \mathrm{~min}$ is the same as those obtained at $1010^{\circ} \mathrm{C}$ annealed for $1 \mathrm{~h}$. This suggests significant savings in the thermal budget of future devices employing these nanocrystals [18]. In most embedded Ge nanocrystal samples, the interplane distances measured from HRTEM images have led us to conclude that the nanostructures possess diamond structure. Due to the electron diffraction patterns, one could say that the Ge nanocrystals of figure 4(c) show typical crystal habits of a diamond structure; these images are very close to the projection of a cuboctahedron, which consists of eight (111) planes and six (100) planes, from the [100] direction.

We have performed a detailed analysis of TEM micrographs for various samples in order to extract information on the diffusion characteristics of $\mathrm{Ge}$ in the $\mathrm{SiO}_{2}$ matrix. The result of this analysis from a two pair sample is displayed in figure 5, where Ge concentration as a function of distance from the $\mathrm{Si}$ substrate interface is shown. The figure demonstrates the precipitation of Ge in layers as a function of the annealing temperature in the ranges of 750 $900{ }^{\circ} \mathrm{C}$. At low temperatures Ge precipitation occurs in the middle of the germanosilicate layers and no bilayer formation is seen. As the annealing temperature increases, broadening of the Ge profiles is observed. At $850^{\circ} \mathrm{C}$, broadening is accompanied by a second hump on each Ge peak on the $\mathrm{Si}$ substrate interface side. At $900^{\circ} \mathrm{C}$, a clear shift of the $\mathrm{Ge}$ profile towards the $\mathrm{Si}$ substrate interface is accompanied by distinctly observable secondary precipitates closer to the interface. A comparison of the total area under the Ge distribution curves for the sample annealed at 750 and $900{ }^{\circ} \mathrm{C}$ indicates that the amount of Ge that precipitated at $750{ }^{\circ} \mathrm{C}$ is not conserved as the annealing temperature is increased. Ge still remaining in the $\mathrm{SiO}_{2}$ : Ge matrix continues to precipitate, accompanied, possibly, by outdiffusion of Ge from the larger size Ge nanocrystals. At this point it is not clear why the additional precipitating band of Ge at higher temperatures does not merge with the initial Ge band broadening it. One possibility is the release of $\mathrm{Ge}$ atoms from $\mathrm{GeOx}$ environments with Si atoms out-diffusing from the substrate [24]. This may explain the precipitation of the second smaller Ge nanocrystal layer closer to the Si substrate interface. However, our observations of the Si substrate crystal planes at the interface do not provide evidence for this possibility. Alternatively, diffusion of Ge atoms aided by increasing stress fields towards the interface may be considered. 


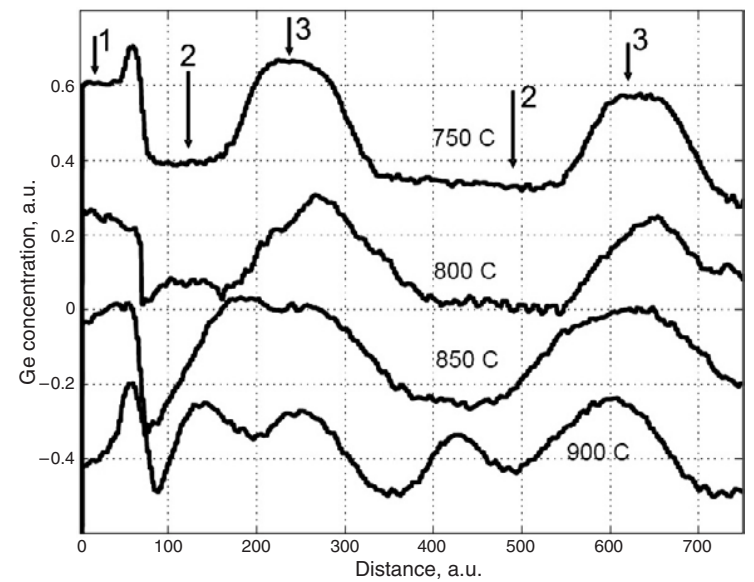

Figure 5. Assuming that the Ge concentration is proportional to the grey scale of the TEM micrograph, Ge concentration profiles have been calculated for several samples grown with $90 \mathrm{sccm}$ germane flow rate and annealed at $750-900^{\circ} \mathrm{C}$ for $5 \mathrm{~min}$. The evolution of the smaller size Ge nanocrystals forming a bilayer is clearly observed at 850 and $900^{\circ} \mathrm{C}$.

The observation of the formation of PECVD grown high quality Ge nanocrystals in multilayers of Ge doped oxide layers is an important result of this work. The separation of Ge nanocrystals with different sizes into two layers is also a crucial result of this study and may be especially important for flash memory applications. Both results suggest that it may be possible to grow multilayered stacks of Ge nanocrystals with controlled separations ranging from a few nanometres to tens of nanometres which may help improve retention times in nanocrystal memory devices. A long retention time will be possible in the doubly stacked nanocrystal memory, since the charge leak between the upper nanocrystals and the channel can be suppressed by an energy barrier due to quantum confinement and Coulomb blockade in the lower nanocrystals. Charge retention will be improved by making the lower nanocrystal size smaller, because the energy barrier is higher in smaller nanocrystals. From electrical measurements it is found that the retention time of the double layer nanocrystal devices over the single layer ones is much improved. The charge transfer prefers direct tunnelling through thin tunnelling and interlayer oxides even in the double layer nanocrystal structure [14]. Further investigation of the dynamics of this bilayer formation may shed light into ways of controlling the size of the nanocrystals.

We have also grown 10 pairs of $\mathrm{SiO}_{2} / \mathrm{SiO}_{2}$ : Ge layers and each one has been separated with about $20 \mathrm{~nm}$ oxide thicknesses. These samples have been grown with germane flow rates of $90 \mathrm{sccm}$ followed by annealing at $800^{\circ} \mathrm{C}$ for $4 \mathrm{~min}$. Cross section TEM image in figure 6(a) shows 10 germanosilicate layers with nanocrystal formation. Here, germanosilicate layers (1), oxide layers (2) and Si substrate (3) are identified. Each period consists of a $20 \mathrm{~nm}$ germanosilicate layer and $40 \mathrm{~nm} \mathrm{SiO} \mathrm{S}_{2}$. We note that bilayer formation for these samples was not observed since the anneal time (4 min) is short and anneal temperature is relatively low $\left(800^{\circ} \mathrm{C}\right)$. Based on an HRTEM image of these samples, figure 6(b) shows Ge nanocrystals (1) with an average size of $8 \mathrm{~nm}$. The micrograph shows perfect alignment of crystallographic planes in the Ge nanocrystals that have clearly pronounced facets. A closer look at different nanocrystals shows that different orientations of crystallographic planes in the Ge nanocrystals are present, indicating the presence of twin boundaries. We conclude that Ge nanocrystals form in each layer on the multilayer sample. 


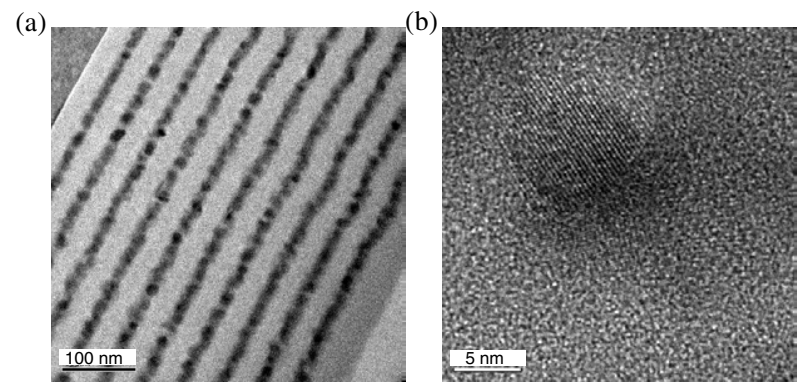

Figure 6. Cross section TEM micrograph of a 10 pair multilayered sample, grown with $90 \mathrm{sccm}$ of germane flow rate annealed at $800^{\circ} \mathrm{C}$ for 4 min exhibiting Ge nanocrystal bands (a), and a high resolution TEM image of the Ge nanocrystals (b). Note that Ge nanocrystal bilayers have not formed due to low temperature of annealing.

\section{Conclusions}

Using HRTEM, EDAX, and electron diffraction we have shown the formation of crystalline Ge nanocrystals in $\mathrm{SiO}_{2}$ matrices grown with the PECVD technique. Each layer in multilayers of $\mathrm{SiO}_{2}: \mathrm{Ge} / \mathrm{SiO}_{2}$ contains Ge nanocrystals. The mean size of the Ge nanocrystals was found to be between 5 and $14 \mathrm{~nm}$ depending on the annealing temperature and duration. With increasing annealing temperature, defect density decreases and crystal sizes increase. At $900{ }^{\circ} \mathrm{C}$, a bilayer of Ge nanocrystals forms with a band of small size Ge nanocrystals and a band of larger size nanocrystals. These Ge nanocrystals embedded in the $\mathrm{SiO}_{2}$ multilayers have potential for application in memory devices. We have found that annealing temperature is a critical parameter for the formation of Ge nanocrystals. The temperature and time duration of annealing determine the size and quality of Ge nanocrystals. Multilayered stacks of Ge nanocrystals with separations ranging from a few nanometres to tens of nanometres can be obtained suitable for future memory devices.

\section{Acknowledgments}

This work is a supported by SEMINANO, a European Union FP6 project and by a Scientific and Technical Research Council of Turkey (TUBITAK) grant, TBAG-85/U. TEM work was carried out at the Center for Microanalysis of Materials, University of Illinois, which is partially supported by the US Department of Energy (DEFG02-91-ER45439). One of us (SA) gratefully acknowledges the financial support of the Scientific and Technical Research Council of Turkey (TUBITAK) to visit UIUC. We thank Dr A Çelik-Aktaş for help with TEM work and Professor J M Zuo for his hospitality. We gratefully acknowledge Professor S Suzer of Bilkent University Chemistry Department for the XPS measurements.

\section{References}

[1] Wang K L, Liu J L and Jin G 2002 J. Cryst. Growth 237-239 1892

[2] Wang Y Q, Kong G L, Chen W D, Diao H W, Chen C Y, Zhang S B and Liao X B 2002 Appl. Phys. Lett. 814174

[3] Averin D V and Likharev K K 1986 J. Low-Temp. Phys. 772394

[4] Meseguer F, Blanco A, Miguez H, Garcia-Santamaria F, Ibisate M and Lopez C 2002 Colloids Surf. 202281

[5] Shlimak I, Vagner I and Safarov V I 2000 Proc. 25th Int. Conf. on the Physics of Semiconductors (Osake: Springer)

[6] Jie Y E, Xiong Y N, Wee A T S, Huan C H A and Ji W 2000 Appl. Phys. Lett. 773936 
[7] Nishii J, Kintaka K, Hosono H, Kawazoe H, Kato M and Muta K 1999 Phys. Rev. B 607166

[8] Kim D W, Prins F E, Kim T, Hwang S, Lee C H, Kwong D L and Banerjee S K 2003 IEEE Trans. Electron Devices $\mathbf{5 0} 510$

[9] Baron T, Pelissier B, Perniola L, Mazen F, Hartmann J M and Rolland G 2003 Appl. Phys. Lett. 831444

[10] De Blauwe J 2002 IEEE Trans. Nanotechnol. 172

[11] Niquet Y, Delerue C, Allan G and Lannoo M 2000 The Fall 2000 MRS Mtg (Boston, MA)

[12] Ohba R, Sugiyama N and Uchida K 2002 IEEE Trans. Electron Devices 491392

[13] Lee C, Gorur-Seetharam A and Kan E C 2003 IEDM Tech. Dig. 557

[14] Maeda M, Yamamoto E, Ohfuji S and Itsumi M 1999 J. Vac. Sci. Technol. B 17201

[15] Serincan U, Kartopu G, Guenness A, Finstad T G, Turan R, Ekinci Y and Bayliss S C 2004 Semicond. Sci. Technol. 19247

[16] Kolobov A V, Wei S Q, Yan W S, Oyanagi H, Maeda Y and Tanaka K 2003 Phys. Rev. B 67195314

[17] Bonafos C, Garrido B, Lopes M, Perez-Rodriguez A, Morante J R, Kihn Y, Ben Assayag G and Claverie A 2000 Appl. Phys. Lett. 763962

[18] Choi W K, Ho V, Ng V, Ho Y W, Ng S P and Chim W K 2005 Appl. Phys. Lett. 86143114

[19] Ağan S, Çelik-Aktaş A, Zuo J M, Dana A and Aydınlı A 2006 Appl. Phys. A 83107

[20] Modreanu M, Aperathitis E, Androulidaki M, Audier M and Chaix-Pluchery O 2005 Opt. Mater. 271020

[21] Photopoulos P, Nassiopoulou A G, Kouvatsos D N and Travlos A 2000 Mater. Sci. Eng. B 69/70 345

[22] Lockwood D J, Lu Z H and Baribeau J M 1996 Phys. Rev. Lett. 76539

[23] Nihonyanagi S, Nishimoto K and Kanemitsu Y 2002 J. Non-Cryst. Solids 299-302 1095

[24] Maeda Y 1995 Phys. Rev. B 511658 


\section{Corrigendum}

TEM studies of nanocrystal formation in PECVD grown for $\mathrm{SiO}_{2}: \mathbf{G e} / \mathrm{SiO}_{2}$ multilayers S Agan 2006 J. Phys.: Condens. Matter 18 5037-5045

In figure 4(c) the diffraction pattern of the $\mathrm{Si}$ substrate was inadvertently included instead of the diffraction pattern of Ge nanocrystals, as shown below:

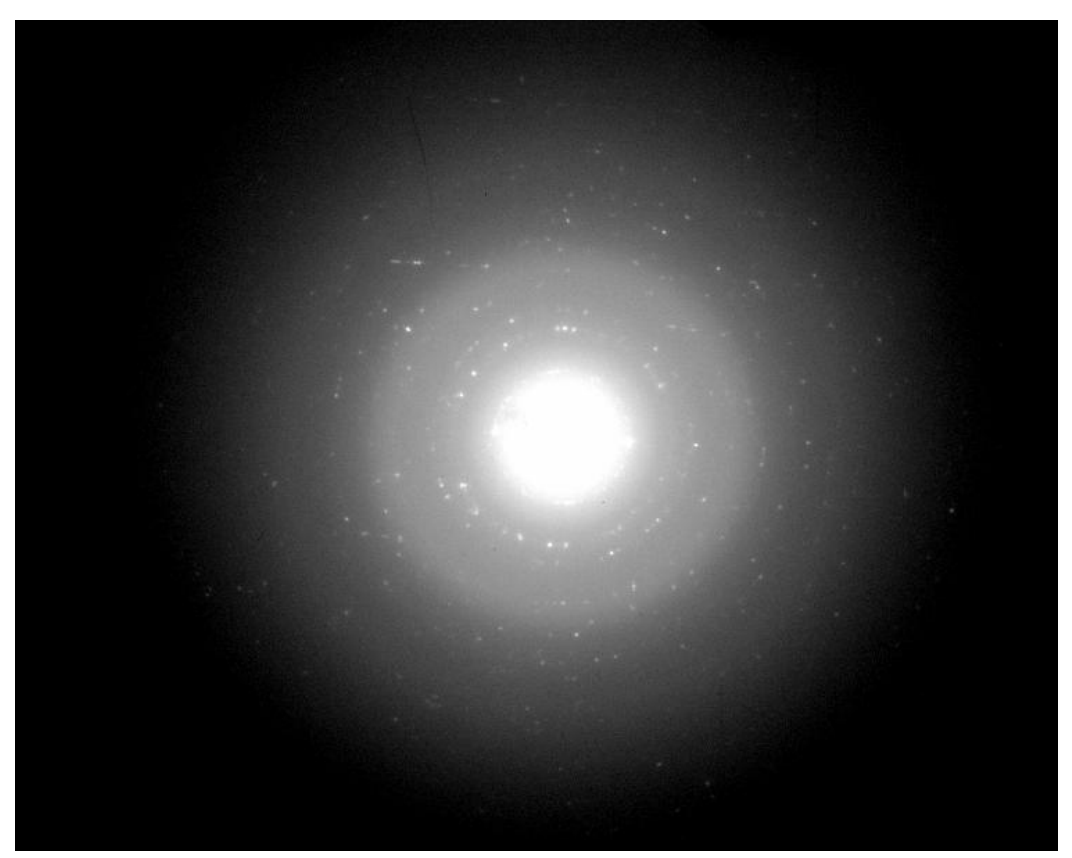

Figure 4(c). Electron beam diffraction pattern of Ge nanocrystals 